\title{
Baseline Liver Function and Subsequent Outcomes in the Phase 3 REFLECT Study of Patients with Unresectable Hepatocellular Carcinoma
}

\author{
Arndt Vogel ${ }^{a}$ Catherine Frenette $^{b}$ Max Sung ${ }^{c}$ Bruno Daniele ${ }^{d}$ Ari Baron $^{e}$ \\ Stephen L. Chan ${ }^{f}$ Jean Frédéric Blanc ${ }^{g}$ Toshiyuki Tamai ${ }^{\text {h }}$ Min Ren ${ }^{\mathrm{i}}$ \\ Howard J. Lim ${ }^{j}$ Daniel H. Palmerk Yuko Takamil Masatoshi Kudom \\ aDepartment of Gastroenterology, Hepatology, and Endocrinology, Hannover Medical School, Hannover, Germany; \\ ${ }^{b}$ Department of Transplantation Hepatology, Scripps MD Anderson Cancer Center, La Jolla, CA, USA; 'Department of \\ Hematology-Oncology, Tisch Cancer Institute at Mount Sinai, New York, NY, USA; ${ }^{d}$ Department of Oncology, Ospedale \\ del Mare, Napoli, Italy; ' Department of Clinical Oncology, Pacific Hematology Oncology Associates, San Francisco, \\ CA, USA; fDepartment of Clinical Oncology, The State Key Laboratory of Translational Oncology, Sir YK Pao Centre for \\ Cancer, The Chinese University of Hong Kong, Hong Kong, China; ${ }^{9}$ Department of Clinical Research and Innovation, \\ University Hospital of Bordeaux, Bordeaux, France; hEisai Co., Ltd., Tokyo, Japan; 'Eisai Inc., Biostatistics, Oncology \\ Business Group, Woodcliff Lake, NJ, USA; 'Department of Medicine, British Columbia Cancer, Vancouver, BC, Canada; \\ kDepartment of Medical Oncology, Liverpool Experimental Cancer Medicine Centre, University of Liverpool, Liverpool, \\ UK; 'Department of Hepato-Biliary-Pancreatic Surgery, National Hospital Organization Kyushu Medical Center, Clinical \\ Research Institute, Fukuoka, Japan; 'mDepartment of Medicine, Kindai University Faculty of Medicine, Osakasayama, \\ Japan
}

\section{Keywords}

Lenvatinib · Sorafenib · Albumin-bilirubin grade ·

Child-Pugh score

\begin{abstract}
Introduction: Baseline liver function among patients starting treatment for unresectable hepatocellular carcinoma (uHCC) impacts survival and could impact efficacy outcomes and safety profiles of treatments. This post hoc analysis of the phase 3 REFLECT study examined the efficacy and safety outcomes for lenvatinib and for sorafenib in patients with uHCC, assessed by Child-Pugh score (CPS) and albumin-bilirubin (ALBI) grade. Methods: Efficacy and safety were assessed in patient cohorts from REFLECT according to study
\end{abstract}

entry baseline ALBI grade and CPS. Results: Lenvatinib treatment generally provided survival benefits in all groups. Median overall survival (OS) among patients with an ALBI grade of 1 was consistently higher than among patients with an ALBI grade of 2 for both the lenvatinib and sorafenib arms (lenvatinib: 17.4 vs. 8.6 months; sorafenib: 14.6 vs. 7.7 months, respectively). Median OS among patients with a CPS of 5 was consistently higher than among patients with a CPS of 6 (lenvatinib: 15.3 vs. 9.4 months; sorafenib: 14.2 vs. 7.9 months, respectively). Progression-free survival and objective response rates for these ALBI grades and CPS demonstrated similar patterns. Among patients who received lenvatinib and experienced a treatment-related treatmentemergent adverse event leading to withdrawal, $6.6 \%$ had an ALBI grade of 1, while $13.3 \%$ had an ALBI grade of 2, and $7.9 \%$ karger@karger.com www.karger.com/lic

Karger $\stackrel{\text { ' }}{5}$

GOPEN ACCESS
(C) 2021 The Author(s)

Published by S. Karger AG, Basel

This is an Open Access article licensed under the Creative Commons Attribution-NonCommercial-4.0 International License (CC BY-NC) (http://www.karger.com/Services/OpenAccessLicense), applicable to the online version of the article only. Usage and distribution for commercial purposes requires written permission.
Correspondence to:

Arndt Vogel, vogel.arndt@mh-hannover.de 
had a CPS of 5, while $12.1 \%$ had a CPS of 6 . Conclusions: Better liver function at baseline, as measured by ALBI grade or CPS, may be prognostic for better survival outcomes in patients with uHCC undergoing treatment with lenvatinib or sorafenib.

(C) 2021 The Author(s).

Published by S. Karger AG, Basel

\section{Introduction}

The incidence of hepatocellular carcinoma (HCC) is increasing in the USA and in many regions around the world [1-3]. HCC also has a high rate of mortality [1-3], making early detection and management particularly important. While its etiology varies worldwide, cirrhosis due to nonalcoholic fatty liver disease, alcohol exposure, hepatitis $\mathrm{B}$, or hepatitis $\mathrm{C}$ is a common cause of HCC [1-3]. Many patients with HCC present with advanced liver disease or cirrhosis and impaired liver function; hence, baseline liver function assessments at diagnosis could provide a potential prognostic indicator in these patients.

Baseline liver function affects survival outcomes in patients with HCC, and several markers of liver function have been identified as independent markers of poor prognosis [4]. Moreover, several liver function scoring assessments have been reported to be prognostic indicators in patients with HCC, including the Cancer of the Liver Italian Program (CLIP) score, Child-Pugh score (CPS), and albumin-bilirubin (ALBI) grade $[5,6]$. The CPS, originally used to estimate mortality risk for patients undergoing surgery for bleeding esophageal varices [7], has since been developed as a prognostic tool for patients with chronic liver disease and cirrhosis. The ALBI grade is a newer liver function assessment that was developed to combat some of the weaknesses of the CPS in patients with HCC - specifically the lack of discrimination among patients who fall into a particular class and the subjectiveness of ascites and encephalopathy measurements [6].

Previously, there were few available options other than sorafenib for the systemic treatment of HCC. In patients treated with sorafenib, baseline liver function was shown to have a prognostic effect. In a study of patients with inoperable liver cancer, sunitinib was found to be significantly inferior to sorafenib [8], but baseline ALBI grade was found to be prognostic for overall survival (OS) in patients who received sorafenib [9]. A prospective study of Japanese patients treated with sorafenib found a prog- nostic relationship between OS and baseline CPS [10]. However, these studies were conducted prior to the approval of lenvatinib for the first-line treatment of patients with unresectable HCC (uHCC), and little is known about the prognostic significance of baseline liver function in patients treated with lenvatinib.

Lenvatinib is a multikinase inhibitor of vascular endothelial growth factor receptors 1-3, fibroblast growth factor receptors 1-4, platelet-derived growth factor receptor a, RET, and KIT [11-14]. In the phase 3, randomized, multicenter, open-label, noninferiority study, REFLECT, lenvatinib demonstrated noninferiority to sorafenib in the first-line treatment of patients with uHCC [15]. Consequently, lenvatinib was approved in multiple countries for the first-line treatment of patients with uHCC [16, 17]. In this post hoc analysis, we assessed the efficacy and safety outcomes for patients in REFLECT based on baseline liver function using the CPS and ALBI grade.

\section{Materials and Methods}

\section{Study Design}

This post hoc retrospective analysis included patients who participated in REFLECT (NCT01761266). Details of the REFLECT methodology have been previously published [15]. For this analysis, efficacy and safety outcomes were analyzed in patient cohorts defined by baseline liver function assessments (i.e., ALBI grade of 1 or 2 , or CPS of 5 or 6 ). Only patients from the ALBI grade 1 or 2 and the CPS of 5 or 6 categories who received lenvatinib or sorafenib were included in this assessment. The CPS was calculated through use of 5 criteria: serum bilirubin, serum albumin, ascites, encephalopathy, and international normalized ratio. Points were assigned to each criterion depending on increasing severity, with the lowest total value (i.e., 5) indicating least hepatic impairment [18]. Only albumin and bilirubin were used to calculate ALBI score using the formula of linear predictor $=\left(\log _{10}\right.$ bilirubin $\times 0.66)+($ albumin $\times-0.085)$, where bilirubin is measured in units of $\mu \mathrm{mol} / \mathrm{L}$ and albumin is in units of $\mathrm{g} / \mathrm{L}$. Scores were then categorized into a grade of $1-3$ as follows: $\leq-2.60$ (grade 1 ), $>-2.60$ and $\leq-1.39$ (grade 2 ), and $>-1.39$ (grade 3 ), with a grade of 1 indicating the least hepatic impairment [6].

\section{Patients}

In REFLECT, 954 patients were randomly assigned, using a 1:1 ratio, to receive either lenvatinib or sorafenib in 28 -day cycles. The starting dose for lenvatinib was based on bodyweight, with patients weighing $\geq 60 \mathrm{~kg}$ receiving $12 \mathrm{mg} /$ day and patients weighing $<60 \mathrm{~kg}$ receiving $8 \mathrm{mg} /$ day. Patients randomly assigned to sorafenib received $400 \mathrm{mg}$ twice daily [15].

Eligible patients were $\geq 18$ years of age and had clinically confirmed uHCC, $\geq 1$ measurable target lesion based on modified Response Evaluation Criteria in Solid Tumors (mRECIST) assessment, Barcelona Clinic Liver Cancer (BCLC) stage B or C, and a CPS of 5 or 6 . Eligible patients also had to have adequate organ function. Adequate hepatic function was defined as albumin 


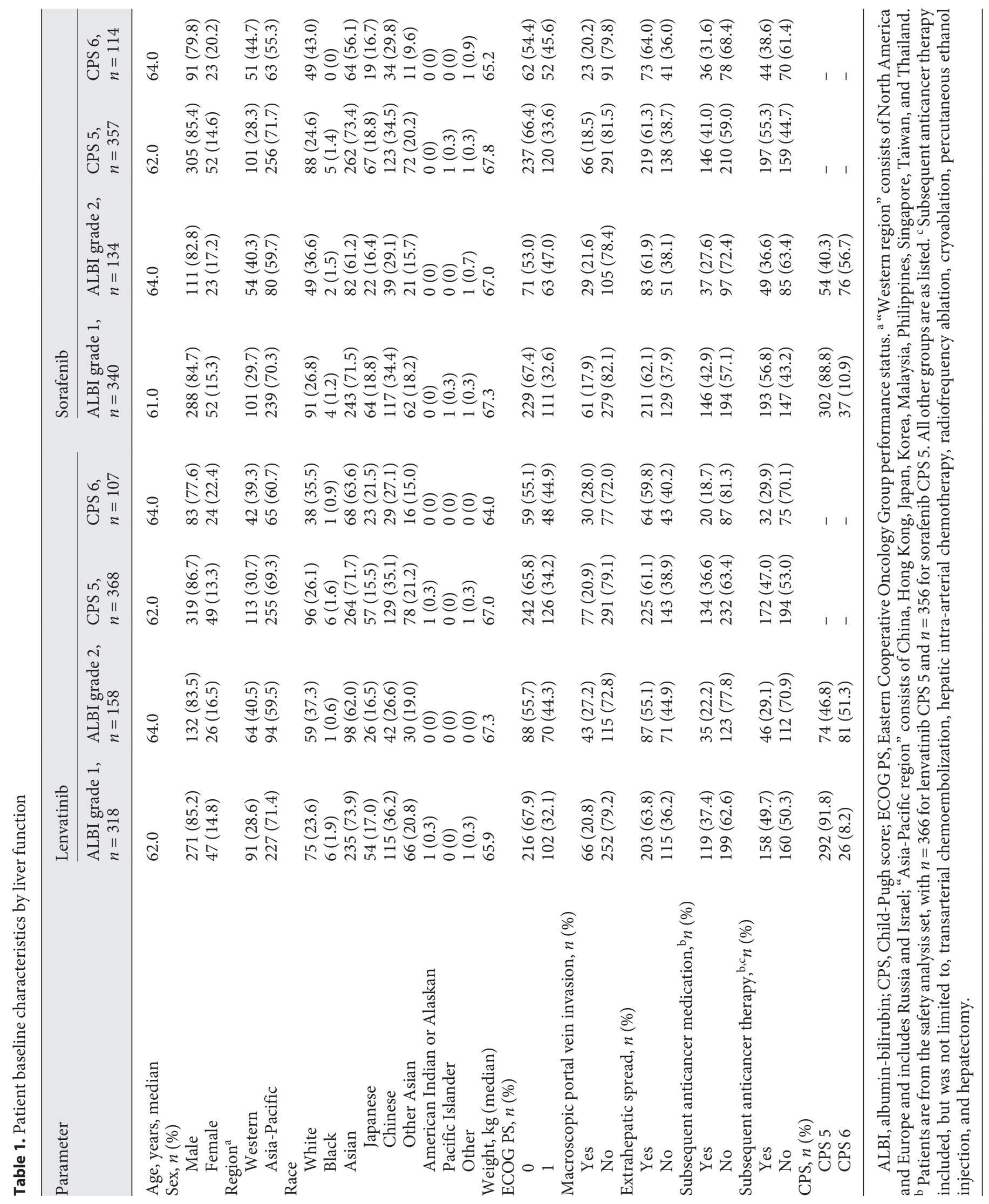


Table 2. Summary of responses by baseline liver function (by IIR using mRECIST)

\begin{tabular}{|c|c|c|c|c|c|c|c|c|}
\hline \multirow[t]{2}{*}{ Response } & \multicolumn{2}{|l|}{ ALBI grade 1} & \multicolumn{2}{|l|}{ ALBI grade 2} & \multicolumn{2}{|l|}{ CPS 5} & \multicolumn{2}{|l|}{ CPS 6} \\
\hline & $\begin{array}{l}\text { Lenvatinib } \\
(n=318)\end{array}$ & $\begin{array}{l}\text { Sorafenib } \\
(n=340)\end{array}$ & $\begin{array}{l}\text { Lenvatinib } \\
(n=158)\end{array}$ & $\begin{array}{l}\text { Sorafenib } \\
(n=134)\end{array}$ & $\begin{array}{l}\text { Lenvatinib } \\
(n=368)\end{array}$ & $\begin{array}{l}\text { Sorafenib } \\
(n=357)\end{array}$ & $\begin{array}{l}\text { Lenvatinib } \\
(n=107)\end{array}$ & $\begin{array}{l}\text { Sorafenib } \\
(n=114)\end{array}$ \\
\hline $\begin{array}{l}\text { Median OS, } \\
\text { months ( } 95 \% \mathrm{CI})\end{array}$ & $17.4(14.1-19.8)$ & $14.6(12.5-16.9)$ & $8.6(7.0-11.5)$ & $7.7(6.1-10.2)$ & $15.3(13.4-18.2)$ & $14.2(12.0-16.3)$ & $9.4(7.0-12.1)$ & $7.9(6.3-11.7)$ \\
\hline $\begin{array}{l}\text { Hazard ratio } \\
(95 \% \text { CI })\end{array}$ & \multicolumn{2}{|c|}{$0.85(0.70-1.02)$} & \multicolumn{2}{|c|}{$0.95(0.73-1.25)$} & \multicolumn{2}{|c|}{$0.91(0.77-1.09)$} & \multicolumn{2}{|c|}{$0.91(0.67-1.24)$} \\
\hline $\begin{array}{l}\text { Median PFS, } \\
\text { months ( } 95 \% \text { CI) }\end{array}$ & $7.4(7.2-9.1)$ & $3.6(3.6-4.1)$ & $5.5(3.6-7.4)$ & $3.5(1.9-3.7)$ & $7.3(5.6-7.6)$ & $3.7(3.6-4.1)$ & $7.4(3.7-9.2)$ & $3.5(1.9-3.7)$ \\
\hline $\begin{array}{l}\text { Hazard ratio } \\
(95 \% \text { CI })\end{array}$ & \multicolumn{2}{|c|}{$0.57(0.47-0.70)$} & \multicolumn{2}{|c|}{$0.76(0.56-1.03)$} & \multicolumn{2}{|c|}{$0.63(0.53-0.76)$} & \multicolumn{2}{|c|}{$0.65(0.45-0.94)$} \\
\hline $\begin{array}{l}\text { ORR, } n(\%) \\
(95 \% \mathrm{CI})\end{array}$ & $143(45.0)(39.5-50.4)$ & $47(13.8)(10.2-17.5)$ & $51(32.3)(25.0-39.6)$ & $12(9.0)(4.1-13.8)$ & $158(42.9)(37.9-48.0)$ & $50(14.0)(10.4-17.6)$ & $36(33.6)(24.7-42.6)$ & $9(7.9)(2.9-12.8)$ \\
\hline
\end{tabular}

ALBI, albumin-bilirubin; CI, confidence interval; CPS, Child-Pugh score; IIR, independent imaging review; mRECIST, modified Response Evaluation Criteria in Solid Tumors; ORR, objective response rate; OS, overall survival; PFS, progression-free survival.

$\geq 2.8 \mathrm{~g} / \mathrm{dL}$ and bilirubin $\leq 3.0 \mathrm{mg} / \mathrm{dL}$, and aspartate aminotransferase, alkaline phosphatase, and alanine aminotransferase levels $\leq 5$ times the upper limit of normal. Patients with previous systemic therapy for HCC and $\geq 50 \%$ liver occupation, bile duct invasion, or portal vein invasion at the main portal branch (Vp4) were excluded. Patient stratification and treatment allocations were based on region (Asia-Pacific or Western); macroscopic portal vein invasion, extrahepatic spread, or both (yes or no); Eastern Cooperative Oncology Group performance status (ECOG PS) (0 or 1); and bodyweight ( $<60$ or $\geq 60 \mathrm{~kg})$ [15].

All patients in the original clinical study provided written informed consent [15]. The study protocol, protocol amendments, and informed consent forms were reviewed and approved by the relevant Institutional Review Boards/independent Ethics Committees. The original study was conducted in accordance with the principles of the Declaration of Helsinki and Good Clinical Practice Guidelines. This post hoc analysis used only data from the original study and did not require Ethics Committee approval.

\section{Endpoints and Clinical Assessments}

Efficacy analyses included OS, progression-free survival (PFS), and objective response rate (ORR). Tumor measurements were performed every 8 weeks by investigator assessment by mRECIST using computed tomography or magnetic resonance imaging until radiologic disease progression, and were also retrospectively assessed by independent imaging review (IIR) using mRECIST; only IIR data are presented here. CPS were assessed at baseline and then at the onset of each 28-day cycle to determine the time to deterioration to Child-Pugh class B. ALBI grade was assessed at baseline, day 15 of the first cycle, days 1 and 15 of the second cycle, and then at the onset of each subsequent cycle. Patients completed an offtreatment visit within 30 days after the last administration of study drug for final assessments. Safety assessments comprised monitoring and recording all treatment-emergent adverse events (TEAEs) using Common Terminology Criteria for Adverse Events v4.0, as well as regular laboratory, electrocardiogram, and vital sign evaluations. Statistical analyses for REFLECT were conducted as previously described [15].

Baseline Liver Function and Outcomes in Patients with uHCC

\section{Results}

\section{Patient Characteristics}

Of the 954 patients enrolled in REFLECT, 478 were randomly assigned to receive lenvatinib and 476 were randomly assigned to receive sorafenib. Of these, $476 \mathrm{pa}-$ tients actually received lenvatinib and 475 received sorafenib. Patient characteristics according to baseline liver function and stratified by ALBI grade and CPS are summarized in Table 1. ALBI grade and CPS seemed to be associated with other prognostic factors, including region, race, and ECOG PS. In general, more patients from the Asia-Pacific region than the Western region had lower ALBI grades and lower CPS at baseline (i.e., ALBI 1 and CPS 5); similarly, patients who identified as Asian had lower ALBI grades and lower CPS at baseline than patients who identified as white. Patients with better baseline liver function were more likely to have an ECOG PS of 0 .

There was considerable overlap between a CPS of 5 and an ALBI grade of 1 . Of patients who received lenvatinib and had an ALBI grade of $1,91.8 \%$ had a CPS of 5; among patients who received sorafenib and had an ALBI grade of $1,88.8 \%$ had a CPS of 5 . Similarly, of patients who received lenvatinib and had a CPS of $5,79.8 \%$ had an ALBI grade of 1 ; among patients who received sorafenib and had a CPS of $5,84.8 \%$ had an ALBI grade of 1 . Fewer patients with an ALBI grade of 1 (66.8\%) and more patients with an ALBI grade of 2 (33.2\%) received lenvatinib compared with sorafenib (ALBI grade 1, 71.6\%; ALBI grade 2, 28.2\%). Patients in either treatment group with an ALBI grade of 1 or a CPS of 5 were more likely to receive subsequent anticancer medication or therapy than patients with an ALBI grade of 2 or a CPS 


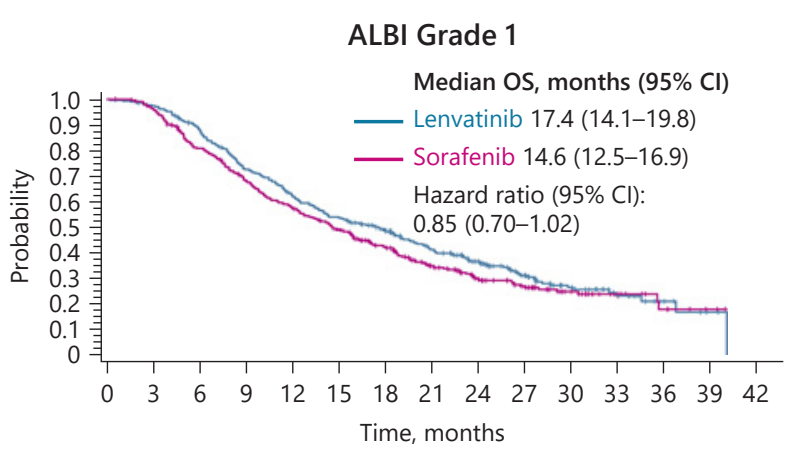

Number of patients at risk:

Lenvatinib $318308276226192166144114 \quad 85 \quad 56 \quad 33 \quad 18 \quad 7 \quad 2 \quad 0$ Sorafenib $340324267223187159128 \quad 94 \quad 67 \quad 46 \quad 26 \quad 13 \quad 6 \quad 2 \quad r$

ALBI Grade 2

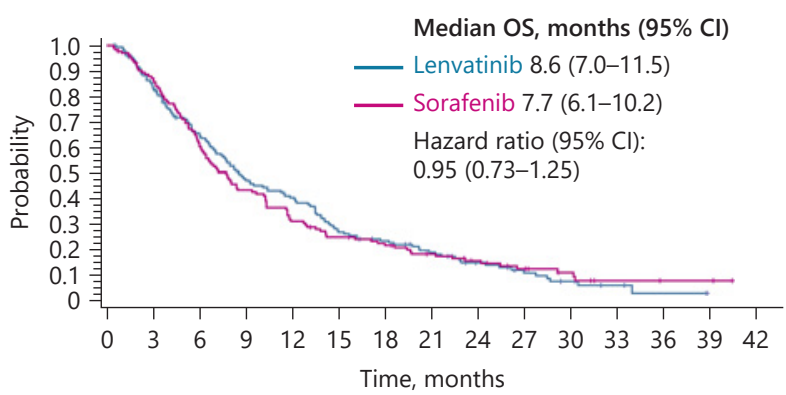

Number of patients at risk:

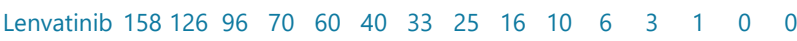

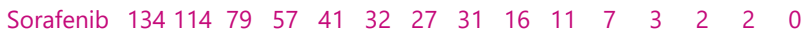

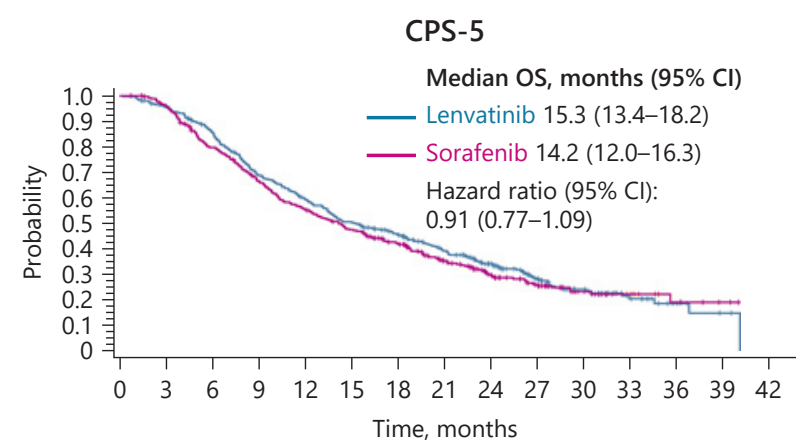

Number of patients at risk:

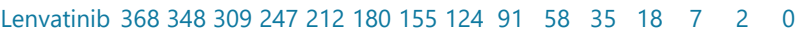

Sorafenib $35734027722919016213410070 \quad 46 \quad 24 \quad 12 \quad 6 \quad 2 \quad 0$

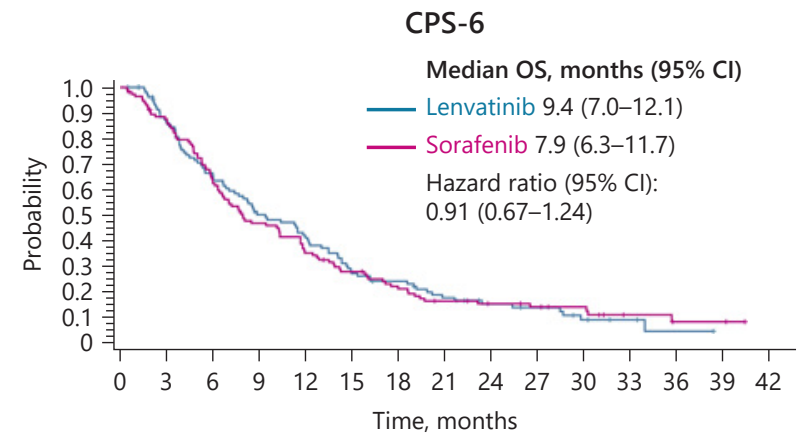

Number of patients at risk:

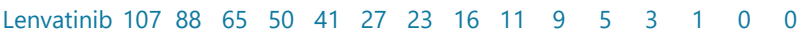

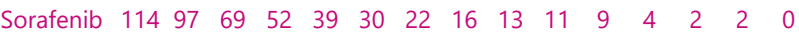

Fig. 1. Kaplan-Meier plots of OS by baseline liver function.

of 6 (Table 1). Similar trends were observed for patients with ALBI grade 1 versus ALBI grade 2, irrespective of whether they had tumor responses with study drug or not (online suppl. Table 1; for all online suppl. material, see www.karger.com/doi/10.1159/000516490).

\section{Efficacy}

Median OS was longer in patients with better baseline liver function as assessed by ALBI grade or CPS (shown in Table 2; Fig. 1). Patients receiving lenvatinib with a baseline ALBI grade of 1 had a median OS of 17.4 months (95\% CI: 14.1-19.8), whereas patients receiving lenvatinib with a baseline ALBI grade of 2 had a median OS of 8.6 months (95\% CI: $7.0-11.5)$. This longer median OS was also seen in patients treated with sorafenib who enrolled with better baseline liver function: the median OS among patients with a base- line ALBI grade of 1 was 14.6 months (95\% CI: $12.5-$ 16.9 ), and among patients with a baseline ALBI grade of 2, it was 7.7 months (95\% CI: 6.1-10.2). Patients with a CPS of 5 had a median OS of 15.3 months (95\% CI: 13.4-18.2) in the lenvatinib arm and 14.2 months (95\% CI: 12.0-16.3) in the sorafenib arm, while patients with a CPS of 6 had a median OS of 9.4 months (95\% CI: 7.0-12.1) in the lenvatinib arm and 7.9 months (95\% CI: 6.3-11.7) in the sorafenib arm. Among patients who received subsequent anticancer therapy, the median OS for patients who had received lenvatinib and had a baseline ALBI grade of $1(n=158)$ was 20.9 months (95\% CI: 17.6-25.9), and for patients who had received lenvatinib and had a baseline ALBI grade of $2(n=46)$, the median OS was 15.1 months (95\% CI: $12.2-20.8$; hazard ratio 0.643 , 95\% CI: $0.440-$ 0.938; data not shown). 


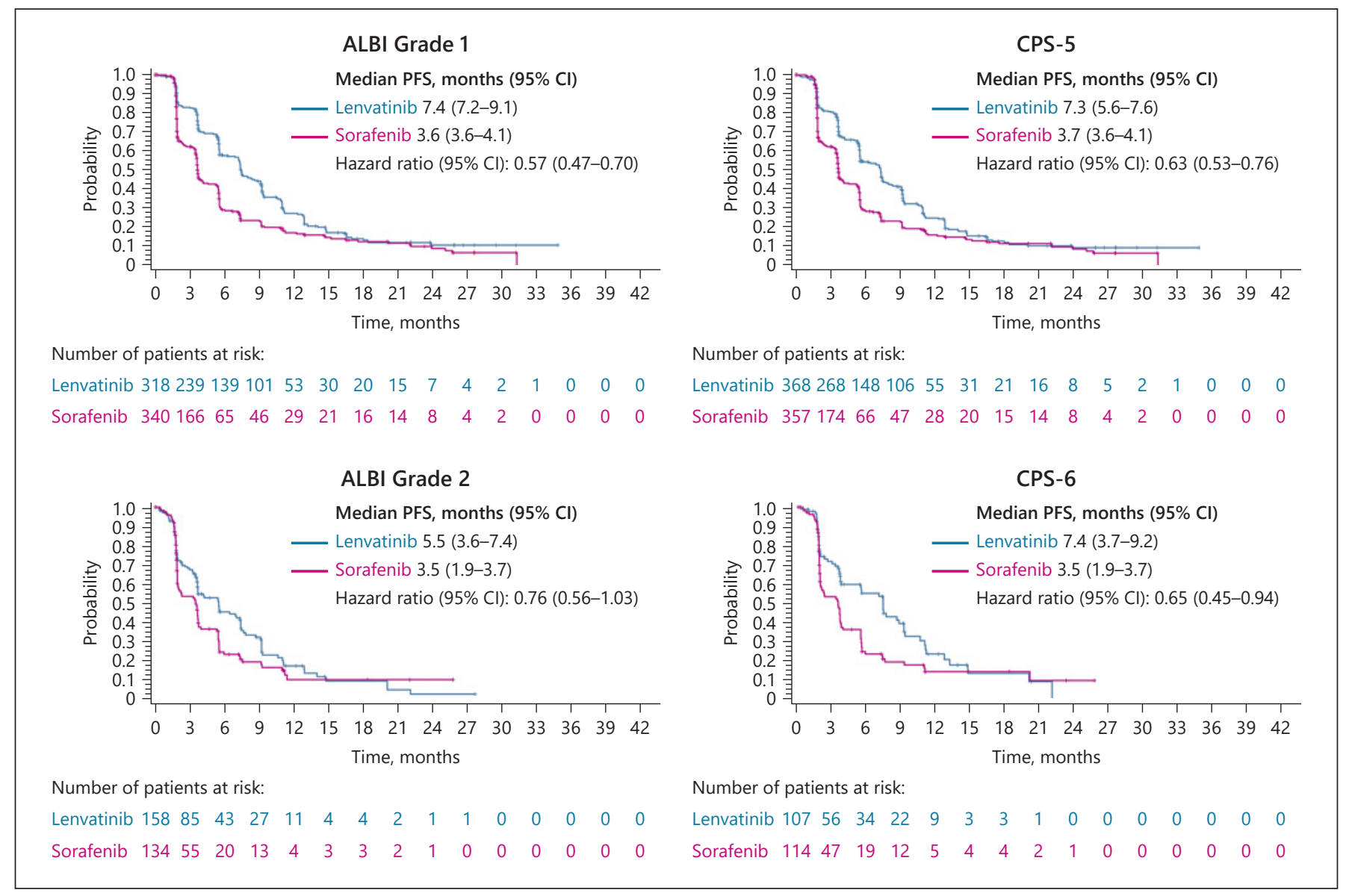

Fig. 2. Kaplan-Meier plots of PFS by baseline liver function (by IIR using mRECIST).

Median PFS, as measured by IIR using mRECIST, was longer in patients who were treated with lenvatinib and had better baseline liver function as measured by ALBI grade (shown in Table 2; Fig. 2). Among patients who received lenvatinib, those with a baseline ALBI grade of 1 had a median PFS of 7.4 months (95\% CI: 7.2-9.1), while patients with a baseline ALBI grade of 2 had a median PFS of 5.5 months (95\% CI: 3.6-7.4). The ALBI grade did not appear to impact PFS in patients receiving sorafenib; patients with a baseline ALBI grade of 1 had a median PFS of 3.6 months (95\% CI: 3.6-4.1) and patients with a baseline ALBI grade of 2 had a median PFS of 3.5 months $(95 \%$ CI: 1.9-3.7). Differences were minimal when measuring PFS according to baseline CPS for both arms. Among patients who received lenvatinib, those with a baseline CPS of 5 had a median PFS of 7.3 months (95\% CI: 5.6-7.6) and patients with a baseline CPS of 6 had a median PFS of 7.4 months (95\% CI: 3.7-9.2). Patients receiving sorafenib with a baseline CPS of 5 had a median PFS of 3.7 months
(95\% CI: 3.6-4.1) and with a baseline CPS of 6 had a median PFS of 3.5 months (95\% CI: 1.9-3.7).

The ORR (assessed by IIR per mRECIST) was higher in patients with better baseline liver function (Table 2). The ORR among patients treated with lenvatinib was $45.0 \%$ for patients with a baseline ALBI grade of 1 and $32.3 \%$ for patients with a baseline ALBI grade of $2 ; 42.9 \%$ for patients with a baseline CPS of 5 and $33.6 \%$ for patients with a baseline CPS of 6 . Among patients treated with sorafenib, the ORR was $13.8 \%$ for patients with a baseline ALBI grade of 1 and $9.0 \%$ for patients with a baseline ALBI grade of 2 , and $14.0 \%$ for patients with a baseline CPS of 5 and $7.9 \%$ for patients with a baseline CPS of 6.

Overall, treatment with lenvatinib provided consistently numerically longer median OS, median PFS, and higher ORR compared with sorafenib, regardless of baseline liver function (Table 2). This survival benefit provided by lenvatinib in groups according to baseline liver 


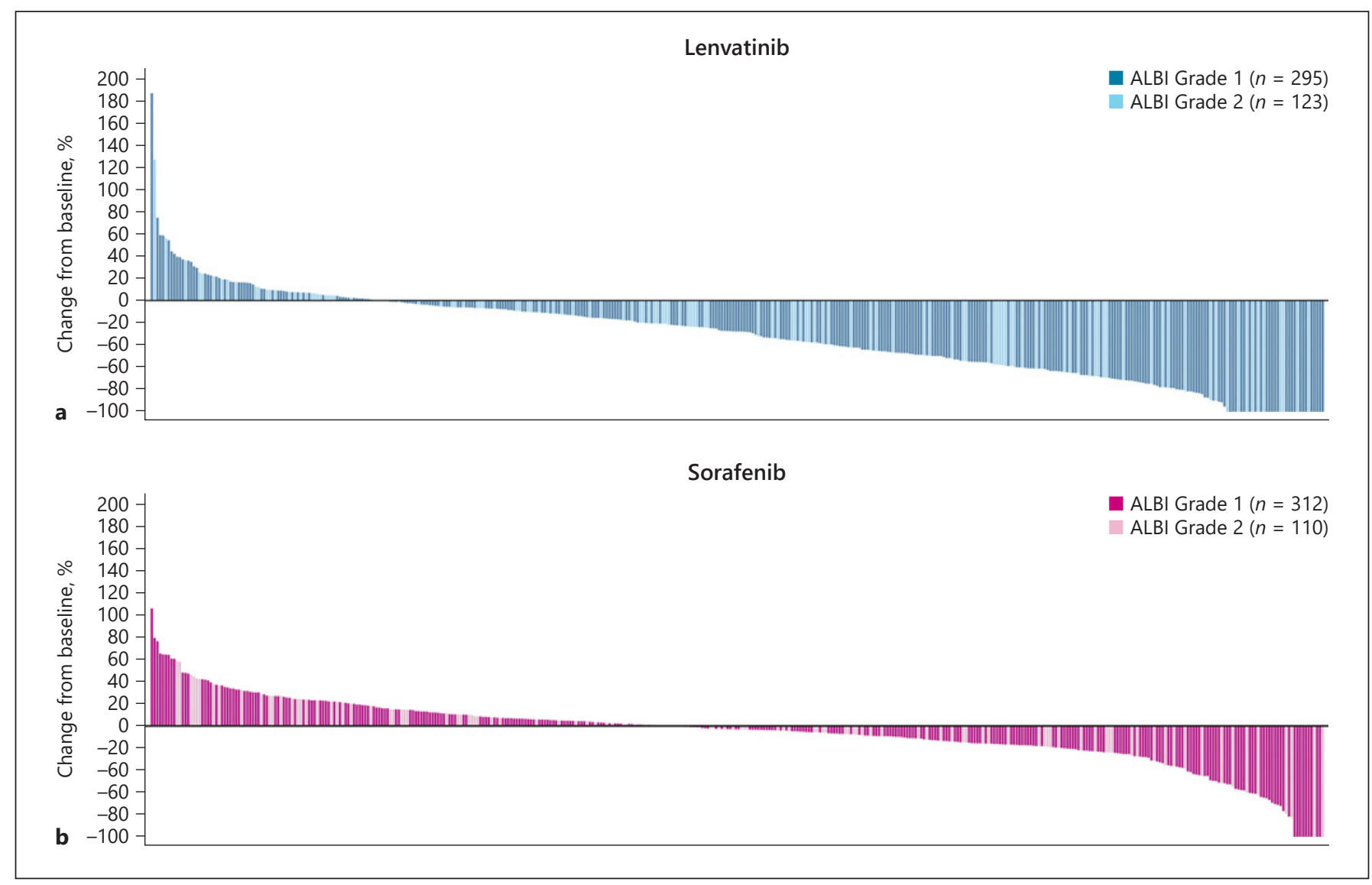

Fig. 3. Percentage changes in the sums of diameters of target lesions at postbaseline nadir for patients treated with lenvatinib (a) or sorafenib (b) (by IIR using mRECIST).

function is consistent with the overall results from REFLECT. Most patients treated with lenvatinib experienced a decrease in tumor diameter at postbaseline nadir (shown in Fig. 3).

While ALBI score remained moderately consistent throughout the study, median values were higher at the off-treatment visit compared with baseline; note, these results should be interpreted with care because patient numbers decreased over time (shown in Fig. 4). At the off-treatment visit, the mean ALBI score for patients who received lenvatinib was -2.46 (grade 2 , standard deviation [SD] 0.60) and the mean ALBI score for patients who received sorafenib was -2.47 (grade $2, \mathrm{SD} 0.62$ ). These values were increases from the means at baseline for patients in both the lenvatinib ( -2.77 , grade $1, S D 0.45)$ and sorafenib (-2.81, grade $1, \mathrm{SD} 0.43$ ) arms (shown in Fig. 4). In patients with a baseline ALBI grade of 1 , a median time to deterioration to Child-Pugh class B was not reached in either treatment arm; however, the rate of deterioration appeared to be slower in patients receiving lenvatinib compared with sorafenib (shown in Fig. 5). No differences were seen among patients with baseline ALBI grade 2 (shown in Fig. 5). Among patients who deteriorated to Child-Pugh class B by week 8 , the median OS was shorter in both treatment arms. Patients who received lenvatinib and declined to Child-Pugh class B by week 8 $(n=57)$ had a median OS of 6.7 months (95\% CI: $2.4-$ 9.7), whereas patients who received lenvatinib and were still in Child-Pugh class A at week $8(n=401)$ had a median OS of 13.3 months (95\% CI: 11.6-16.1). Patients who received sorafenib and declined to Child-Pugh class B by week $8(n=43)$ had a median OS of 4.5 months ( $95 \%$ CI: 2.9-6.1), whereas patients who received sorafenib and were still in Child-Pugh class A at week $8(n=416)$ had a median OS of 12.0 months (95\% CI: 10.2-14.0) (data not shown). 


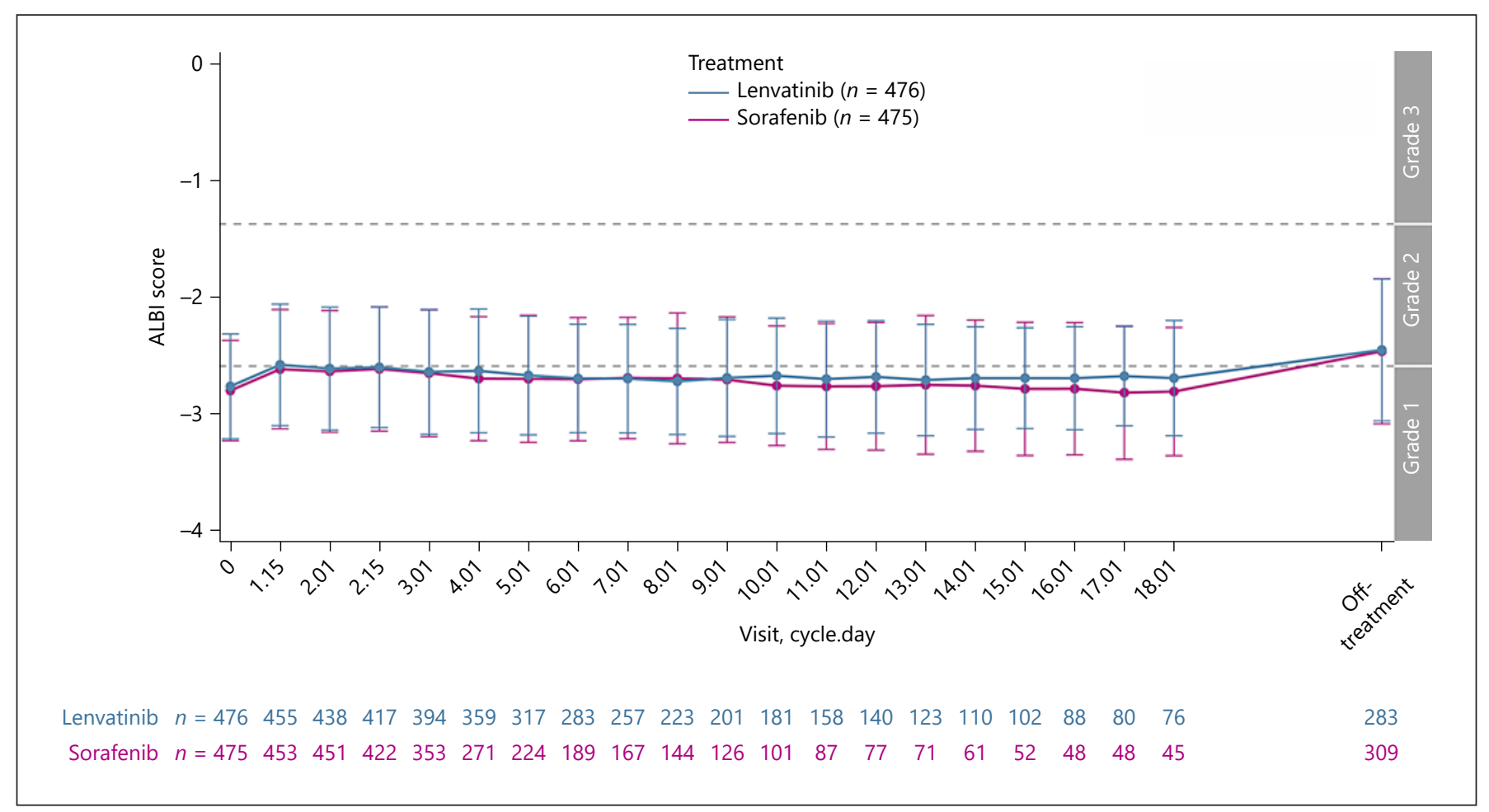

Fig. 4. Median ALBI score over time.

\section{Safety}

With lenvatinib treatment, rates of TEAEs grade $\geq 3$ were lower in patients with better baseline liver function (i.e., lower ALBI grade or CPS) (shown in Table 3). Among patients with a baseline ALBI grade of 1,69.5\% experienced at least 1 grade $\geq 3$ TEAE versus $86.1 \%$ of patients with a baseline ALBI grade of 2. Similarly, $71.6 \%$ of patients with a baseline CPS of 5 experienced at least 1 grade $\geq 3$ TEAE versus $86.0 \%$ of patients with a baseline CPS of 6 . The pattern was similar for patients receiving sorafenib: $62.1 \%$ of patients with a baseline ALBI grade of 1 and $63.2 \%$ of patients with a baseline CPS of 5 experienced at least 1 grade $\geq 3$ TEAE, while $77.6 \%$ of patients with a baseline ALBI grade of 2 and $76.3 \%$ of patients with a baseline CPS of 6 experienced at least 1 grade $\geq 3$ TEAE.

Treatment-related TEAEs leading to study drug withdrawal generally occurred less frequently in patients with better baseline liver function (shown in Table 3). Lenvatinib treatment was withdrawn in $6.6 \%$ of patients with a baseline ALBI grade of 1 versus $13.3 \%$ of patients with a baseline ALBI grade of 2, and in $7.9 \%$ of those with a baseline CPS of 5 versus $12.1 \%$ of patients with a baseline CPS of 6. Sorafenib treatment was withdrawn in 7.1\% of patients with a baseline ALBI grade of 1 versus $7.5 \%$ of patients with a baseline ALBI grade of 2, and in $6.5 \%$ of patients with a baseline CPS of 5 versus $9.6 \%$ of patients with a CPS of 6.

Treatment-related TEAEs led to dose reductions at similar rates irrespective of baseline liver function (shown in Table 3). Treatment-related TEAEs led to lenvatinib dose reduction in $35.5 \%$ of patients with a baseline ALBI grade of 1 versus $39.9 \%$ of patients with a baseline ALBI grade of 2 , and in $36.6 \%$ of those with a baseline CPS of 5 versus $39.3 \%$ with a baseline CPS of 6 . Treatment-related TEAEs led to sorafenib dose reduction in $37.1 \%$ of patients with a baseline ALBI grade of 1 versus $40.3 \%$ of patients with a baseline ALBI grade of 2, and in 37.9\% of those with a baseline CPS of 5 versus $38.6 \%$ with a baseline CPS of 6.

Median duration of lenvatinib treatment was longer in patients with better baseline liver function (shown in Table 3). Median duration of lenvatinib treatment was 7.4 months for patients with a baseline ALBI grade of 1 and 6.9 months for patients with a baseline CPS of 5, compared with 3.6 months for patients with a baseline ALBI grade of 2 and 3.7 months for patients with a baseline CPS of 6 . Among patients who received sorafenib, the median duration of treatment was 3.7 months for patients with a 


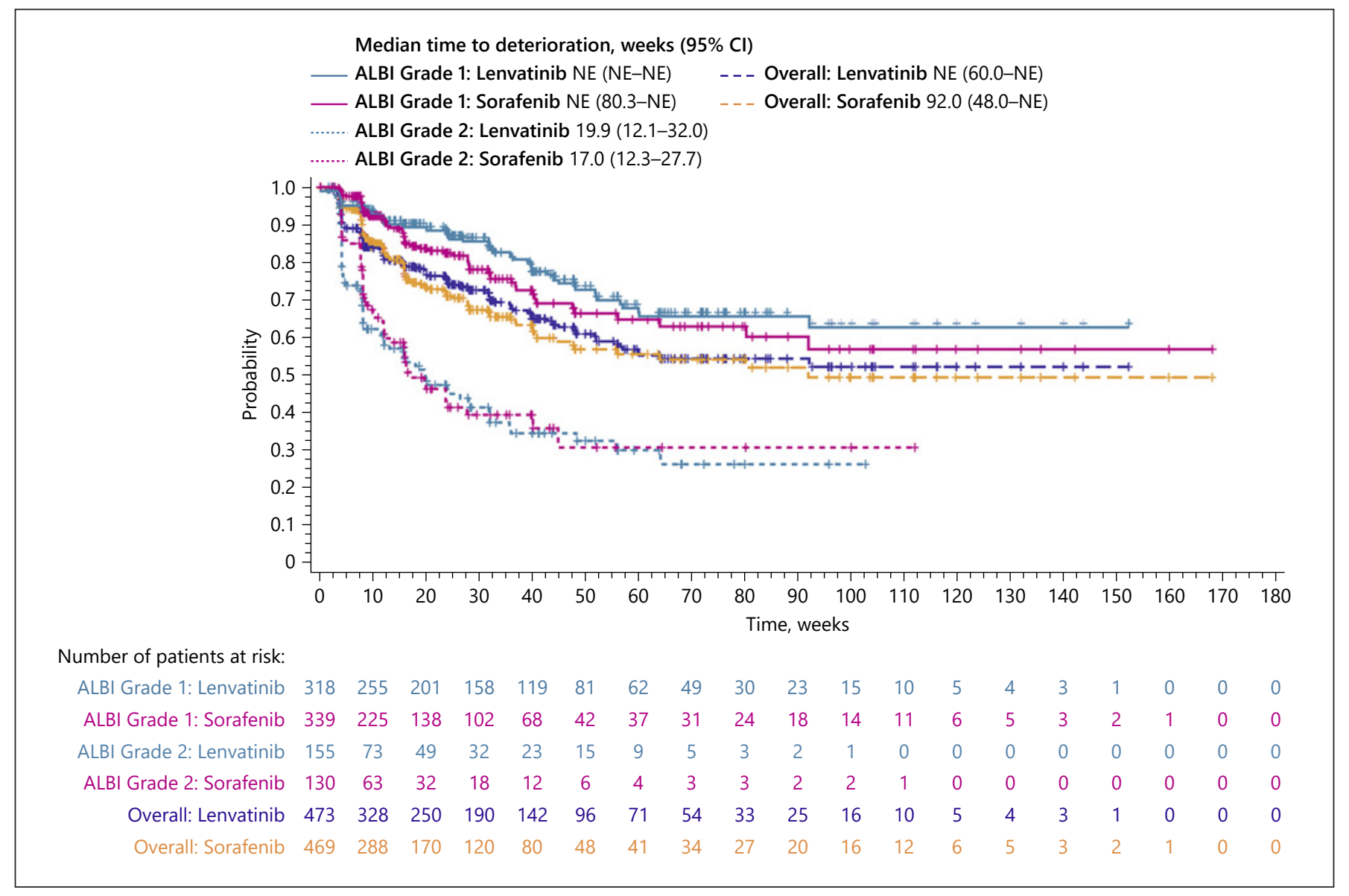

Fig. 5. Time to deterioration to Child-Pugh class $B^{a}$ in ALBI grade 1, ALBI grade 2 , and overall patient populations. ${ }^{\text {a }}$ Time to deterioration from baseline Child-Pugh class to Child-Pugh class B was determined by CPS assessed at baseline, day 1 of cycle 2 to end of treatment, and the off-treatment visit. ALBI, albumin-bilirubin; NE, not estimable.

baseline ALBI grade of 1 and 3.7 months for patients with a baseline CPS of 5, compared with 3.1 months for patients with a baseline ALBI grade of 2 and 3.3 months for patients with a baseline CPS of 6 . Minimal changes were seen in median relative dose intensities across groups based on baseline liver function (shown in Table 3).

\section{Discussion}

This retrospective analysis suggests that a better baseline ALBI grade (for OS, PFS, and ORR) or CPS (for ORR) may be prognostic for better efficacy with lenvatinib or sorafenib treatment in patients with uHCC. This post hoc analysis of data from REFLECT demonstrated that median OS was longer for patients with a baseline ALBI grade of 1 or a baseline CPS of 5 than for patients with a baseline ALBI grade of 2 or a baseline CPS of 6 in patients treated with lenvatinib or sorafenib. Regardless of baseline liver function, numerically greater efficacy was seen with lenvatinib compared with sorafenib as assessed by OS, PFS, and ORR, consistent with the overall results of REFLECT.

Baseline liver function may also be linked to safety outcomes in patients with uHCC who are treated with lenvatinib or sorafenib. Patients in this analysis who had better baseline liver function and who received either lenvatinib or sorafenib experienced fewer grade $\geq 3$ TEAEs. Similarly, patients who received lenvatinib and who had a lower baseline ALBI grade or CPS also required fewer study drug withdrawals and had longer durations of treatment. Overall, in SELECT, TEAEs were generally tolerable, irrespective of baseline ALBI grade or CPS, and toxicity can generally be managed in most patients with dose reductions. 
Table 3. TEAEs, dose modifications, and study drug exposure by baseline liver function

\begin{tabular}{|c|c|c|c|c|c|c|c|c|}
\hline Parameter & $\begin{array}{l}\text { ALBI grade } 1 \\
n=318\end{array}$ & $\begin{array}{l}\text { ALBI grade } 2 \\
n=158\end{array}$ & $\begin{array}{l}\text { CPS 5, } \\
n=366\end{array}$ & $\begin{array}{l}\text { CPS 6, } \\
n=107\end{array}$ & $\begin{array}{l}\text { ALBI grade } 1, \\
n=340\end{array}$ & $\begin{array}{l}\text { ALBI grade } 2 \\
n=134\end{array}$ & $\begin{array}{l}\text { CPS 5, } \\
n=356\end{array}$ & $\begin{array}{l}\text { CPS 6, } \\
n=114\end{array}$ \\
\hline TEAEs grade $\geq 3, n(\%)$ & $221(69.5)$ & $136(86.1)$ & $262(71.6)$ & $92(86.0)$ & $211(62.1)$ & $104(77.6)$ & $225(63.2)$ & $87(76.3)$ \\
\hline \multicolumn{9}{|l|}{ Treatment-related TEAEs leading to } \\
\hline Study drug withdrawal, $n(\%)$ & $21(6.6)$ & $21(13.3)$ & $29(7.9)$ & $13(12.1)$ & $24(7.1)$ & $10(7.5)$ & $23(6.5)$ & $11(9.6)$ \\
\hline Median relative dose intensity, \% & 98.5 & 96.2 & 98.2 & 96.0 & 97.2 & 94.2 & 96.4 & 96.7 \\
\hline
\end{tabular}

ALBI, albumin-bilirubin; CPS, Child-Pugh score; TEAE, treatment-emergent adverse event.

Results for OS are consistent with those reported in other publications for treatment with tyrosine kinase inhibitors. ALBI grade was found to be prognostic for OS in patients with UHCC who were treated with sorafenib or placebo in a retrospective analysis of the SHARP and Asia-Pacific phase 3 clinical trials [19]. Another retrospective analysis of the SHARP trial revealed that sorafenib was effective in patients compared with placebo, though patients with elevated hepatic markers had shorter OS and a decreased disease control rate [4]. A secondary analysis of the sorafenib arm of a phase 3 trial found that an ALBI grade of 1 was significantly associated with better OS [9]. In the phase 3 CELESTIAL trial comparing cabozantinib to placebo in patients with HCC, outcomes were better in patients with ALBI grade 1 compared with ALBI grade 2 [20]. Patients with ALBI grade 1 in the REACH trial were more likely to gain a survival benefit from ramucirumab treatment than patients with ALBI grade 2 or 3 [21]. Together, these results suggest that tyrosine kinase inhibitors are an important component in the sequential systemic treatment of HCC. This current analysis of lenvatinib in uHCC is particularly timely and important because of the ongoing trials of lenvatinib and pembrolizumab combination treatment for HCC [22].

The connection between increased efficacy and better baseline liver status could be linked to several factors. Patients with better liver function may experience longer exposure to treatment, providing greater benefit. Poor liver function in some patients might adversely impact the metabolism of study drugs. The impact of cirrhosis on the liver may also be a competing risk for death. Finally, both tumor progression and drug toxicity could cause liver function to decrease over the course of treatment.

Baseline Liver Function and Outcomes in Patients with uHCC
Preservation of liver function is key for determining the sequence for administration of systemic therapies; as such, liver function should be considered during selection of these therapies. Patients often have better liver function at the beginning of their HCC treatment, which can impact decision-making in multiple treatment scenarios. Specifically, patients who have BCLC stage B or C liver function could be initially administered systemic therapy with the intent to downstage the cancer, and local therapy could be provided subsequently. Further, patients who have undergone transarterial chemoembolization and did not experience a substantial response (over $50 \%$ ) could be switched from local therapy to a systemic therapy to avoid deterioration of liver function.

There are a variety of recommended options for sequential systemic therapy for patients with advanced HCC, according to the European Society for Medical Oncology [23]. Their guidelines recommend sorafenib, lenvatinib, or the combination of atezolizumab and bevacizumab for first-line systemic therapy. Moreover, for patients who progress during first-line therapy, tyrosine kinase inhibitors can be considered for secondline therapy [23]. However, most of these recommendations are for patients with Child-Pugh class A, and limited data are available for those patients with worse liver function at baseline. Because the ALBI grading system is more granular, it allows for more discrimination among patients within Child-Pugh class A and is therefore a valuable assessment tool.

This analysis was limited by the post hoc nature of the assessment. Additionally, the Child-Pugh system has a number of limitations [6]. The Child-Pugh system is a composite score based on 5 parameters including encephalopathy, ascites, serum bilirubin, serum albumin, and international normalized ratio [18]. The grading of

Liver Cancer 2021;10:510-521 519 
some of these parameters (specifically ascites and encephalopathy) can be subjective, leading to discrepancies in scoring among physicians. Further, upon Child-Pugh assessment, most HCC patients are categorized into the Child-Pugh class A, and therefore, limited discrimination among patient status is provided [6]. The ALBI grade attempts to address these issues by providing a liver function assessment that is based upon laboratory values of albumin and bilirubin and is therefore less subjective and easier to generate. Use of this model provided discrimination between ALBI groups that was at least as good as that between Child-Pugh classes when assessing OS in HCC patients in international centers [6]. Moreover, within the Child-Pugh class A, there are 2 distinct prognostic groups with a clear impact on survival, falling under ALBI grade 1 or 2. Recently, modifications of the ALBI grade system have been suggested $[24,25]$. Although precise cutoff values have varied, these reports showed that dividing ALBI grade 2 into $2 \mathrm{a}$ and $2 \mathrm{~b}$ provided even more precise categorization of patients $[24,25]$.

The results of this post hoc analysis of REFLECT based on baseline liver function support the use of the ALBI grade as a prognostic tool. In this analysis, the ALBI grade demonstrated similar prognostic utility to the CPS in efficacy and safety measurements and avoided the subjectivity of the CPS. Therefore, this metric may provide a more attractive method of liver assessment for clinicians because of the requirement for fewer laboratory tests, its probable consistency across providers, and greater ease in measuring change of liver function over time. Although Child-Pugh class A is typically used as a treatment or clinical study criterion, the class is broad and encompasses distinct prognostic groups (i.e., scores 5 and 6 or ALBI grades 1 and 2). As such, an ALBI grade provides a more specific prognostic option.

\section{Conclusions}

Assessment of baseline liver function, including ALBI grade, could provide data to support treatment decisions, particularly the use of repeat locoregional therapies that run the risk of worsening liver function as liver function deteriorates because of treatment toxicity or tumor progression. This assessment could potentially lead toward improved outcomes for patients. Ultimately, to achieve better efficacy with systemic lenvatinib or sorafenib treatment, it may be important to treat patients before baseline liver function deteriorates. The results of this analysis indicate that patients with CPS 6 or
ALBI grade 2 had shorter OS, lower ORR, faster deterioration of liver function, shorter treatment duration, and higher rates of adverse events compared with patients who have better baseline liver function. However, as there is limited clinical research in patients with HCC and Child-Pugh class B liver function, further research is needed to determine the absolute benefit of lenvatinib or sorafenib in patients with Child-Pugh class B baseline liver function.

\section{Statement of Ethics}

The original source study was conducted ethically in accordance with the World Medical Association Declaration of Helsinki. Patients have given their written informed consent, and the study protocol was approved by the institute's Committee on $\mathrm{Hu}$ man Research. However, for this post hoc analysis using only data from the original study, ethics approval was not required.

\section{Conflict of Interest Statement}

A.V. has provided a speaker, consultancy, and advisory role for Roche, Bayer, Sanofi, BMS, Lilly, Novartis, Eisai, AstraZeneca, Merck, Incyte, Medac, Ipsen, Servier, PierreFabre, MSD, BTG, and Janssen. C.F. serves on speakers' bureaus and advisory boards for Bayer and Eisai. M.S. has received honoraria from advisory board participation from Bayer, Eisai, Exelixis, and Genentech. B.D. has received personal fees and nonfinancial support for consultation from Ipsen, Sanofi, and Bayer; and personal fees from Roche, Eisai, Eli Lilly, AstraZeneca, MSD, and Incyte. A.B. has served on speakers' bureau for Bristol Myers Squibb, Merck, Lilly, Amgen, Eisai, Johnson \& Johnson, and AbbVie. S.L.C. has acted as an advisor to AstraZeneca, Eisai, Ipsen, and MSD. J.F.B. has received honoraria from Bayer, Ipsen, Eisai, Roche, BMS, AstraZeneca, and Eli Lilly. T.T. is an employee of and has stock/other ownership with Eisai. M.R. is an employee of Eisai. H.J.L. has nothing to disclose. D.H.P. has received honoraria from Eisai and research funding and honoraria from Bayer, BMS, Roche, AstraZeneca, Sirtex, and BTG. Y.T. has nothing to disclose. M.K. has received honoraria from Merck Sharp \& Dohme, Eisai, Bayer, Lilly Japan, EA Pharma, and Bristol Myers Squibb Japan; served in a consulting/advisory role for Merck Sharp \& Dohme, Eisai, Ono Pharmaceuticals, Bristol Myers Squibb, and Roche; and received research funding (inst) from Otsuka Pharmaceutical, Taiho Pharmaceutical, AbbVie, Takeda Pharmaceuticals, Eisai, Gilead Sciences, EA Pharma, and Dainippon Sumitomo Pharma. M.K. is the Editor-in-Chief of Liver Cancer.

\section{Funding Sources}

This study was funded by Eisai Inc., Woodcliff Lake, NJ, USA, and also by Merck Sharp \& Dohme Corp., a subsidiary of Merck \& Co., Inc., Kenilworth, NJ, USA. Medical writing support was pro- 
vided by Heather A. Mitchell of Oxford PharmaGenesis Inc., Newtown, PA, USA, and was funded by Eisai Inc., Woodcliff Lake, NJ, USA, and also by Merck Sharp \& Dohme Corp., a subsidiary of Merck \& Co., Inc., Kenilworth, NJ, USA.

\section{References}

1 Fujiwara N, Friedman SL, Goossens N, Hoshida Y. Risk factors and prevention of hepatocellular carcinoma in the era of precision medicine. J Hepatol. 2018;68(3):526-49.

2 Massarweh NN, El-Serag HB. Epidemiology of hepatocellular carcinoma and intrahepatic cholangiocarcinoma. Cancer Control. 2017; 24(3):1073274817729245.

3 Mittal S, El-Serag HB. Epidemiology of hepatocellular carcinoma: consider the population. J Clin Gastroenterol. 2013;47(Suppl): S2-6.

4 Raoul JL, Bruix J, Greten TF, Sherman M, Mazzaferro V, Hilgard P, et al. Relationship between baseline hepatic status and outcome, and effect of sorafenib on liver function: SHARP trial subanalyses. J Hepatol. 2012; 56(5):1080-8.

5 The Cancer of the Liver Italian Program (CLIP) Investigators. Prospective validation of the CLIP score: a new prognostic system for patients with cirrhosis and hepatocellular carcinoma. The Cancer of the Liver Italian Program (CLIP) Investigators. Hepatology. 2000; 31(4):840-5.

6 Johnson PJ, Berhane S, Kagebayashi C, Satomura S, Teng M, Reeves HL, et al. Assessment of liver function in patients with hepatocellular carcinoma: a new evidence-based approach-the ALBI grade. J Clin Oncol. 2015; 33(6):550-8.

7 Child CG, Turcotte JG. Surgery and portal hypertension. Major Probl Clin Surg. 1964;1: $1-85$.

8 Cheng AL, Kang YK, Lin DY, Park JW, Kudo $M$, Qin S, et al. Sunitinib versus sorafenib in advanced hepatocellular cancer: results of a randomized phase III trial. J Clin Oncol. 2013; 31(32):4067-75.

9 Abdel-Rahman O. Impact of baseline characteristics on outcomes of advanced HCC patients treated with sorafenib: a secondary analysis of a phase III study. J Cancer Res Clin Oncol. 2018;144(5):901-8.

10 Ogasawara S, Chiba T, Ooka Y, Kanogawa N, Saito T, Motoyama T, et al. Sorafenib treat-

\section{Author Contributions}

Study design: A.V. Data acquisition: S.L.C., M.S., and D.H.P. Data analysis: M.S. Data interpretation: all authors. Drafting the manuscript, technical review and/or revision, review of the final draft, and approval to submit: all authors. ment in Child-Pugh A and B patients with advanced hepatocellular carcinoma: safety, efficacy and prognostic factors. Invest $\mathrm{New}$ Drugs. 2015;33(3):729-39.

11 Matsui J, Funahashi Y, Uenaka T, Watanabe T, Tsuruoka A, Asada M. Multi-kinase inhibitor E7080 suppresses lymph node and lung metastases of human mammary breast tumor MDA-MB-231 via inhibition of vascular endothelial growth factor-receptor (VEGF-R) 2 and VEGF-R3 kinase. Clin Cancer Res. 2008; 14(17):5459-65.

12 Matsui J, Yamamoto Y, Funahashi Y, Tsuruoka $\mathrm{A}$, Watanabe $\mathrm{T}$, Wakabayashi $\mathrm{T}$, et al. E7080, a novel inhibitor that targets multiple kinases, has potent antitumor activities against stem cell factor producing human small cell lung cancer H146, based on angiogenesis inhibition. Int J Cancer. 2008;122(3):664-71.

13 Tohyama O, Matsui J, Kodama K, Hata-Sugi N, Kimura T, Okamoto K, et al. Antitumor activity of lenvatinib (e7080): an angiogenesis inhibitor that targets multiple receptor tyrosine kinases in preclinical human thyroid cancer models. J Thyroid Res. 2014;2014: 638747.

14 Yamamoto Y, Matsui J, Matsushima T, Obaishi H, Miyazaki K, Nakamura K, et al. Lenvatinib, an angiogenesis inhibitor targeting VEGFR/FGFR, shows broad antitumor activity in human tumor xenograft models associated with microvessel density and pericyte coverage. Vasc Cell. 2014;6:18.

15 Kudo M, Finn RS, Qin S, Han KH, Ikeda K, Piscaglia F, et al. Lenvatinib versus sorafenib in first-line treatment of patients with unresectable hepatocellular carcinoma: a randomised phase 3 non-inferiority trial. Lancet. 2018;391(10126):1163-73.

16 Eisai Inc. Lenvima (lenvatinib) [prescribing information]. Woodcliff Lake, NJ, USA, Eisai Inc; 2020.

17 Personeni N, Pressiani T, Rimassa L. Lenvatinib for the treatment of unresectable hepatocellular carcinoma: evidence to date. J Hepatocell Carcinoma. 2019;6:31-9.
18 Tsoris A, Marlar CA. Use of the Child Pugh Score in liver disease. Treasure Island, FL: StatPearls; 2020.

19 Bruix J, Cheng AL, Meinhardt G, Nakajima K, De Sanctis Y, Llovet J. Prognostic factors and predictors of sorafenib benefit in patients with hepatocellular carcinoma: analysis of two phase III studies. J Hepatol. 2017;67(5): 999-1008.

20 Miksad R, Cicin I, Chen Y, Klumpen H, Kim $\mathrm{S}$, Lin Z, et al. Outcomes based on AlbuminBilirubin (ALBI) grade in the phase 3 CELESTIAL trial of cabozantinib versus placebo in patients with advanced hepatocellular carcinoma (HCC) [abstract]. Ann Oncol. 2019; 30(Suppl 4):iv134. Abstract O-022.

21 Blanc J-F, Chan SL, Park JO, Ryoo B-Y, Yen C-J, Kudo M, et al. Ramucirumab as secondline treatment in patients with advanced hepatocellular carcinoma: analysis of REACH patients by albumin-bilirubin (ALBI) grade [abstract]. J Hepatol. 2016;64:S693-4. Abstract SAT-076.

22 Finn RS, Ikeda M, Zhu AX, Sung MW, Baron $\mathrm{AD}$, Kudo M, et al. Phase Ib study of lenvatinib plus pembrolizumab in patients with unresectable hepatocellular carcinoma. J Clin Oncol. 2020;38(26):2960-70.

23 Vogel A, Cervantes A, Chau I, Daniele B, Llovet JM, Meyer T, et al. Hepatocellular carcinoma: ESMO Clinical Practice Guidelines for diagnosis, treatment and follow-up. Ann Oncol. 2018;29(Suppl 4):iv238-iv55.

24 Hiraoka A, Kumada T, Atsukawa M, Hirooka M, Tsuji K, Ishikawa T, et al. Prognostic factor of lenvatinib for unresectable hepatocellular carcinoma in real-world conditions-multicenter analysis. Cancer Med. 2019;8(8):371928.

25 Ogasawara S, Chiba T, Ooka Y, Suzuki E, Kanogawa N, Saito T, et al. Liver function assessment according to the Albumin-Bilirubin (ALBI) grade in sorafenib-treated patients with advanced hepatocellular carcinoma. Invest New Drugs. 2015;33(6):125762. 